\title{
On the Lower Classes of Some Mixed Fractional Gaussian Processes with Two Logarithmic Factors
}

\author{
Charles El-Nouty \\ U.F.R. de Mathématiques, Université Pierre et Marie Curie, 175 rue du Chevaleret, 75013 Paris, France \\ Correspondence should be addressed to Charles El-Nouty, elnouty@ccr.jussieu.fr \\ Received 5 February 2007; Revised 10 July 2007; Accepted 12 September 2007 \\ Recommended by Hideo Nagai \\ We introduce the fractional mixed fractional Brownian sheet and investigate the small ball behavior \\ of its sup-norm statistic by establishing a general result on the small ball probability of the sum of \\ two not necessarily independent joint Gaussian random vectors. Then, we state general conditions \\ and characterize the sufficiency part of the lower classes of some statistics of the above process by \\ an integral test. Finally, when we consider the sup-norm statistic, the necessity part is given by a \\ second integral test.
}

Copyright (C) 2008 Charles El-Nouty. This is an open access article distributed under the Creative Commons Attribution License, which permits unrestricted use, distribution, and reproduction in any medium, provided the original work is properly cited.

\section{Introduction}

Let $\left\{B_{H_{i}}(s), s \geq 0\right\}$ be a fractional Brownian motion (FBM) with index $0<H_{i}<1, i \in \mathbb{N}^{*}$, and $\left\{B_{H_{j}, H_{k}}\left(s, s^{\prime}\right), s \geq 0, s^{\prime} \geq 0\right\}$ a fractional Brownian sheet (FBS) with index $0<H_{j}, H_{k}<1, j \in$ $\mathbb{N}^{*}, k \in \mathbb{N}^{*}$. We refer to [1] for further information about the FBM and the FBS. Denote by $\lambda_{1}$ and $\lambda_{2}$ two real numbers such that $\lambda_{1} \lambda_{2} \neq 0$.

Define a fractional mixed fractional Gaussian process by a suitable combination of some appropriate fractional Gaussian processes. In the sequel, we consider the following three examples.

Example 1.1. The fractional mixed fractional Brownian motion (FMFBM) is defined by

$$
X\left(w_{1}, w_{2}, s\right)=\lambda_{1} s^{H_{2}} B_{H_{1}}\left(w_{1}\right)+\lambda_{2} s^{H_{1}} B_{H_{2}}\left(w_{2}\right),
$$

where $B_{H_{1}}$ and $B_{H_{2}}$ are independent FBM with $H_{1} \neq H_{2}$. 
Example 1.2. The fractional mixed fractional Brownian motion and fractional Brownian sheet (FMFBMFBS) are defined by

$$
X\left(w_{1}, w_{2}, w_{3}, s\right)=\lambda_{1} s^{H_{2}+H_{3}} B_{H_{1}}\left(w_{1}\right)+\lambda_{2} s^{H_{1}} B_{H_{2}, H_{3}}\left(w_{2}, w_{3}\right),
$$

where the FBM $B_{H_{1}}$ and the FBS $B_{H_{2}, H_{3}}$ are independent.

Example 1.3. The fractional mixed fractional Brownian sheet (FMFBS) is defined by

$$
X\left(w_{1}, w_{2}, w_{3}, w_{4}, s\right)=\lambda_{1} s^{H_{3}+H_{4}} B_{H_{1}, H_{2}}\left(w_{1}, w_{2}\right)+\lambda_{2} s^{H_{1}+H_{2}} B_{H_{3}, H_{4}}\left(w_{3}, w_{4}\right),
$$

where $B_{H_{1}, H_{2}}$ and $B_{H_{3}, H_{4}}$ are independent FBS with $\left(H_{1}, H_{2}\right) \neq\left(H_{3}, H_{4}\right)$.

The motivation supporting this paper is threefold.

(i) The first goal of the FMFBS deals with the potential applications. Since the FMFBM, the FMFBMFBS, and the FMFBS can be analyzed based on the large bodies of knowledge on FBM and FBS, it can be used in the same fields, that is, natural time series in economics, fluctuations in solid, hydrology, and, more recently, by new problems in mathematical finance, telecommunication networks, and the environment (see [2-4]).

(ii) A second application deals with the small ball probability problem of the sum of two not necessarily joint centered Gaussian random vectors $X$ and $Y$ in a separable Banach space $E$ with norm $\|\cdot\|$ (see [5]). The small ball behavior of the FMFBS under the uniform norm can be investigated as a special case of the small ball probability problem of the sum of two centered Gaussian random vectors, having a log-type small ball factor (see [6]).

(iii) Last but not least, this article extends El-Nouty's results [6-9] and consequently answers some new questions. Recall first two definitions of the Lévy classes, stated in [10]. Let $\{Z(t), t \geq 0\}$ be a stochastic process defined on the basic probability space $(\Omega, \AA)$.

Definition 1.4. The function $f(t), t \geq 0$, belongs to the lower-lower class of the process $Z,(f \in$ $\operatorname{LLC}(Z))$, if, for almost all $\omega \in \Omega$, there exists $t_{0}=t_{0}(\omega)$ such that $Z(t) \geq f(t)$ for every $t>t_{0}$.

Definition 1.5. The function $f(t), t \geq 0$, belongs to the lower-upper class of the process $Z,(f \in$ $\operatorname{LUC}(Z))$, if, for almost all $\omega \in \Omega$, there exists a sequence $0<t_{1}=t_{1}(\omega)<t_{2}=t_{2}(\omega)<\cdots$ with $t_{n} \rightarrow+\infty$, as $n \rightarrow+\infty$, such that $Z\left(t_{n}\right) \leq f\left(t_{n}\right), n \in \mathbb{N}^{*}$.

In the spirit of $[6-9,11]$, the main aim of this paper is to characterize the lower classes of the uniform norm of the FMFBS for any $0<H_{1}, H_{2}, H_{3}, H_{4}<1$. More precisely, we want to compare the influence of two FBSs and to measure the weight of a log-type small ball factor versus another one. 


\section{Main results}

Our first result is given in the following theorem.

Theorem 2.1. Let $X$ and $Y$ be any two joint Gaussian random vectors in a separable Banach space with norm $\|\cdot\|$. Assume that there exist $C_{X} \geq 1$ and $C_{Y} \geq 1$ such that one has, for any $\epsilon>0$ small enough,

$$
\begin{aligned}
& -C_{X} \leq \frac{\epsilon^{1 / \alpha}}{(\log (1 / \epsilon))^{\beta}} \log \mathbb{P}(\|X\| \leq \epsilon) \leq-\frac{1}{C_{X}}, \\
& -C_{Y} \leq \frac{\epsilon^{1 / \tilde{\alpha}}}{(\log (1 / \epsilon))^{\tilde{\beta}}} \log \mathbb{P}(\|Y\| \leq \epsilon) \leq-\frac{1}{C_{Y}},
\end{aligned}
$$

with $0<\alpha, \tilde{\alpha}<+\infty, 0 \leq \beta, \tilde{\beta}<+\infty$ and $(\alpha, \beta) \neq(\tilde{\alpha}, \tilde{\beta})$.

If $(\alpha<\tilde{\alpha})$ or $(\alpha=\tilde{\alpha}$ and $\beta>\tilde{\beta})$, then there exists $K_{X} \geq C_{X}$ depending on $C_{X}$ only such that one has, for any $\epsilon>0$ small enough,

$$
-K_{X} \leq \frac{\epsilon^{1 / \alpha}}{(\log (1 / \epsilon))^{\beta}} \log \mathbb{P}(\|X+Y\| \leq \epsilon) \leq-\frac{1}{K_{X}} .
$$

Since the study of the lower classes of the FMFBM (resp., FMFBMFBS) under the uniform norm was investigated in [8] (resp., [9]), we focus our attention to the FMFBS. Set

$$
Y(t)=\sup _{0 \leq s \leq t} \sup _{0 \leq w_{1}, w_{2}, w_{3}, w_{4} \leq s}\left|X\left(w_{1}, w_{2}, w_{3}, w_{4}, s\right)\right|, \quad t \geq 0 .
$$

Note first that, by the scaling property, we have, for any $\epsilon>0$,

$$
\begin{aligned}
\mathbb{P}\left(Y(t) \leq \epsilon t^{H_{1}+H_{2}+H_{3}+H_{4}}\right) & =\mathbb{P}\left(\sup _{0 \leq s \leq 1} \sup _{0 \leq w_{1}, w_{2}, w_{3}, w_{4} \leq s}\left|X\left(w_{1}, w_{2}, w_{3}, w_{4}, s\right)\right| \leq \epsilon\right) \\
& =\mathbb{P}(Y(1) \leq \epsilon):=\phi(\epsilon),
\end{aligned}
$$

where $\phi$ is named the small ball function and $\gamma:=H_{1}+H_{2}+H_{3}+H_{4}$ the scaling factor.

Recall that the small ball behavior of the FBS under the uniform norm was studied in $[12,13]$.

Set $\alpha=\min \left(H_{1}, H_{2}, H_{3}, H_{4}\right)$, which is in ]0,1[. We introduce the number $\beta$ taking its values in $\{0,1+1 / \alpha\}$. As a direct consequence of Theorem 2.1, we have the following corollary.

Corollary 2.2. There is a constant $K_{0}, 0<K_{0} \leq 1$, depending on $H_{1}, H_{2}, H_{3}, H_{4}, \lambda_{1}$ and $\lambda_{2}$ only, such that one has, for any $\epsilon>0$ small enough,

$$
\exp \left(-\frac{(\log (1 / \epsilon))^{\beta}}{K_{0} \epsilon^{1 / \alpha}}\right) \leq \phi(\epsilon) \leq \exp \left(-\frac{K_{0}(\log (1 / \epsilon))^{\beta}}{\epsilon^{1 / \alpha}}\right) .
$$

Recall that we suppose $\left(H_{1}, H_{2}\right) \neq\left(H_{3}, H_{4}\right)$. In the sequel, there is no loss of generality to assume also that

$$
H_{1} \leq H_{2}, \quad H_{3} \leq H_{4} .
$$


Thus when $\left(H_{1}=H_{2}, H_{3}<H_{4}\right.$ and $\left.H_{1} \leq H_{3}\right),\left(H_{1}=H_{2}, H_{3}=H_{4}\right.$ and $\left.H_{1}<H_{3}\right)$, or $\left(H_{1}<H_{2}, H_{3}=H_{4}\right.$ and $\left.H_{3} \leq H_{1}\right)$, we emphasize that $\beta=1+1 / \alpha$, that is, we have a log-type small ball factor.

Note first that the minimum $\alpha$ plays a key role. This is not really surprising. Indeed, this phenomenon was already observed in $[8,9]$.

It appears that the sufficiency part of the lower classes of $Y$ can be stated in a general framework. Roughly speaking, we follow the same lines as those of $[6,7]$.

Let $\left\{Y_{0}(t), t \geq 0\right\}$ be a real-valued statistic of the two independent FBSs, $B_{H_{1}, H_{2}}$ and $B_{H_{3}, H_{4}}$, such that $Y_{0}(t)$ is a nondecreasing function of $t \geq 0$.

The following notation is needed. If $\mathbb{K}$ is a Hausdorff compact space, we denote, by $C(\mathbb{K})$, the space of all continuous functions from $\mathbb{K}$ to $\mathbb{R}$ equipped with the classical sup-norm. Let $\mathbb{X}=C\left([0,1]^{2}\right) \times C\left([0,1]^{2}\right)$ be the product space equipped with the product topology. Denote, by $\mathrm{L}\left(B_{H_{1}, H_{2}}, B_{H_{3}, H_{4}}\right)$, the Gaussian measure associated to $B_{H_{1}, H_{2}}$ and $B_{H_{3}, H_{4}}$ and defined on $\mathbb{B}$, the Borel $\sigma$-field of $\mathbb{X}$.

We assume that $Y_{0}$ satisfies the following three conditions:

(i) The scaling condition. There exists $\gamma_{0}>0$ such that

$$
\mathbb{P}\left(Y_{0}(t) \leq \epsilon t^{\gamma_{0}}\right)=\mathbb{P}\left(Y_{0}(1) \leq \epsilon\right):=\phi(\epsilon)
$$

(ii) The convexity condition. There exists a convex and $\mathbb{B}$-measurable function $g:(\mathbb{X}, \mathrm{L} \times$ $\left.\left(B_{H_{1}, H_{2}}, B_{H_{3}, H_{4}}\right)\right) \rightarrow \mathbb{R}$ such that, for any $t \geq 0, Y_{0}(t)=g\left(B_{H_{1}, H_{2}}\left(s_{1} t, s_{2} t\right)\right.$, $\left.B_{H_{3}, H_{4}}\left(s_{3} t, s_{4} t\right) ; 0 \leq s_{1}, s_{2}, s_{3}, s_{4} \leq 1\right)$, and $Y_{0}(t)<+\infty$, with probability 1 .

(iii) The log-type small ball condition. There exist $\left.\left.\alpha_{0} \in\right] 0, \gamma_{0}\right], \beta_{0} \in \mathbb{R}$ and a constant $K, 0<$ $K \leq 1$, depending on $H_{1}, H_{2}, H_{3}, H_{4}, \gamma_{0}, \alpha_{0}$ and $\beta_{0}$ only such that we have, for any $\epsilon>0$ small enough,

$$
\exp \left(-\frac{(\log (1 / \epsilon))^{\beta_{0}}}{K \epsilon^{1 / \alpha_{0}}}\right) \leq \phi(\epsilon) \leq \exp \left(-\frac{K(\log (1 / \epsilon))^{\beta_{0}}}{\epsilon^{1 / \alpha_{0}}}\right)
$$

Note that these conditions generalize those of $[6,7]$. The small ball function still plays a key role. The convexity of the function $\psi$ defined by $\psi(\epsilon)=-\log \phi(\epsilon), 0<\epsilon<1$, is ensured by (ii) (see $[14,15])$.

Our second result is given in the following theorem.

Theorem 2.3. Let $f(t)$ be a positive nondecreasing function of $t \geq 0$. Assume that there exists $m>0$ such that $\left(f(t) / t^{r_{0}-\alpha_{0}}\right)\left(\log t^{r_{0}} / f(t)\right)^{-\beta_{0} \alpha_{0}} \geq m$.

If

$$
\frac{f(t)}{t^{\gamma_{0}}} \text { is bounded and } \int_{0}^{+\infty} f(t)^{-1 / \alpha_{0}} t^{\left(\gamma_{0} / \alpha_{0}\right)-1}\left(\log \frac{t^{\gamma_{0}}}{f(t)}\right)^{\beta_{0}} \phi\left(\frac{f(t)}{t^{\gamma_{0}}}\right) d t<+\infty \text {, }
$$


then one has

$$
f \in \operatorname{LLC}\left(Y_{0}\right)
$$

The sup-norm statistic $Y$ clearly satisfies the three above conditions with $\gamma_{0}=\gamma=H_{1}+$ $H_{2}+H_{3}+H_{4}, \alpha_{0}=\alpha=\min \left(H_{1}, H_{2}, H_{3}, H_{4}\right)=\min \left(H_{1}, H_{3}\right), \beta_{0}=\beta \in\{0,1+1 / \alpha\}$, and $K=K_{0}$. Now, we characterize the necessity part of the lower classes of the FMFBMFBS. Our main result is stated in the following theorem.

Theorem 2.4. Let $f(t)$ be a positive nondecreasing function of $t \geq 0$ such that $f(t) / t^{r}$ is a nonincreasing function of $t>0$.

If

$$
f \in L L C(Y)
$$

then one has

$$
\lim _{t \rightarrow+\infty} \frac{f(t)}{t^{\gamma}}=0, \quad \int_{0}^{+\infty} f(t)^{-1 / \gamma} \phi\left(\frac{f(t)}{t^{\gamma}}\right) d t<+\infty
$$

First, we can notice that Theorem 2.3 involves $\gamma_{0}, \alpha_{0}$ and $\beta_{0}$. If $\beta_{0}=0$, Theorem 2.3 looks like [7, Theorem 1] or else like [6, Theorem 1.1]. Theorem 2.4 has the same form as the necessity part of [11, Theorem 1.2] or as the theorems obtained in [6-9]. In his previous works on the study of the lower classes, the author showed that the methodology in [11] led to two integral tests, these tests are actually identical when $\gamma=\alpha$ and $\beta=0$. Here, $\alpha=\min \left(H_{1}, H_{3}\right)<\gamma=$ $H_{1}+H_{2}+H_{3}+H_{4}$. This is why the integral tests of Theorems 2.3 and 2.4 have different forms. Moreover, since $\alpha<\gamma$, we must assume, as in [6-9], that $f(t) / t^{\gamma}$ is not only bounded, but also a nonincreasing function of $t>0$. This last assumption will play a key role in some proofs. Finally, although they are two different integral tests, Theorems 2.3 and 2.4 are sharp. Indeed, set, if $\beta=0$,

$$
f(t)=\frac{\lambda t^{\gamma}}{(\log \log t)^{\alpha}}, \quad t \geq 3, \lambda>0
$$

or else (i.e., $\beta=1+(1 / \alpha)$ )

$$
f(t)=t^{\gamma} \frac{(\lambda \log \log \log t)^{1+\alpha}}{(\log \log t)^{\alpha}}, \quad t \geq 16, \lambda>0 .
$$

If $\lambda$ is small enough, then Theorem 2.3 yields $f \in \operatorname{LLC}(Y)$, or else if $\lambda$ is large enough, then $f \in \operatorname{LUC}(Y)$ by applying Theorem 2.4.

In Section 3, we prove Theorem 2.1. The proof of Theorem 2.4 is postponed to Sections 4 and 5. In the latter, we establish some key small ball estimates. Note also that these estimates can be of independent interest. The proofs, which are modifications of those of [6,7], will be consequently omitted, in particular, the proof of Theorem 2.3. 


\section{Proof of Theorem 2.1}

Recall, first, a Gaussian correlation inequality stated in [5].

Theorem A. Let $\mu$ be a centered Gaussian measure on a separable Banach space E. Then for any $0<$ $\lambda<1$, and any symmetric convex sets $A$ and $B$ in $E$,

$$
\mu(A \cap B) \mu\left(\lambda^{2} A+\left(1-\lambda^{2}\right) B\right) \geq \mu(\lambda A) \mu\left(\left(1-\lambda^{2}\right)^{1 / 2} B\right) .
$$

In particular,

$$
\mu(A \cap B) \geq \mu(\lambda A) \mu\left(\left(1-\lambda^{2}\right)^{1 / 2} B\right) .
$$

Rougly speaking, the proof follows the same lines as those in [15] and will be split into two parts: the lower bound and the upper one.

Part I. The lower bound

Theorem A implies, for any $0<\delta<1$ and $0<\lambda<1$,

$$
\begin{aligned}
\mathbb{P}(\|X+Y\| \leq \epsilon) & \geq \mathbb{P}(\|X\| \leq(1-\delta) \epsilon,\|Y\| \leq \delta \epsilon) \\
& \geq \mathbb{P}(\|X\| \leq \lambda(1-\delta) \epsilon) \mathbb{P}\left(\|Y\| \leq\left(1-\lambda^{2}\right)^{1 / 2} \delta \epsilon\right) .
\end{aligned}
$$

Then we get, by using (2.1),

$$
\log \mathbb{P}(\|X+Y\| \leq \epsilon) \geq-C_{X} \frac{(\log (1 / \lambda(1-\delta) \epsilon))^{\beta}}{(\lambda(1-\delta) \epsilon)^{1 / \alpha}}-C_{Y} \frac{\left(\log \left(1 /\left(1-\lambda^{2}\right)^{1 / 2} \delta \epsilon\right)\right)^{\tilde{\beta}}}{\left(\left(1-\lambda^{2}\right)^{1 / 2} \delta \epsilon\right)^{1 / \tilde{\alpha}}}
$$

Hence, since $(\alpha<\tilde{\alpha})$ or $(\alpha=\tilde{\alpha}$ and $\beta>\widetilde{\beta})$, there exists $C_{X}^{\prime} \geq C_{X}$ depending on $C_{X}$ only such that we have, for any $\epsilon>0$ small enough,

$$
\frac{\epsilon^{1 / \alpha}}{(\log (1 / \epsilon))^{\beta}} \log \mathbb{P}(\|X+Y\| \leq \epsilon) \geq-\frac{C_{X}^{\prime}}{(\lambda(1-\delta))^{1 / \alpha}}
$$

and the lower bound follows by taking $\delta \rightarrow 0$ and $\lambda \rightarrow 1$.

Part II. The upper bound

A new use of Theorem A implies, for any $0<\delta<1$ and $0<\lambda<1$,

$$
\begin{aligned}
\mathbb{P}\left(\|X\| \leq \frac{\epsilon}{(1-\delta) \lambda}\right) & \geq \mathbb{P}\left(\|X+Y\| \leq \frac{\epsilon}{\lambda^{\prime}},\|Y\| \leq \frac{\delta \epsilon}{(1-\delta) \lambda}\right) \\
& \geq \mathbb{P}(\|X+Y\| \leq \epsilon) \mathbb{P}\left(\|Y\| \leq\left(1-\lambda^{2}\right)^{1 / 2} \frac{\delta \epsilon}{(1-\delta) \lambda}\right) .
\end{aligned}
$$

Then combining (2.1) with the fact that $(\alpha<\tilde{\alpha})$ or $(\alpha=\tilde{\alpha}$ and $\beta>\widetilde{\beta})$, there exists $C_{X}^{\prime \prime} \geq C_{X}$ depending on $C_{X}$ only such that we have, for any $\epsilon>0$ small enough,

$$
\frac{\epsilon^{1 / \alpha}}{(\log (1 / \epsilon))^{\beta}} \log \mathbb{P}(\|X+Y\| \leq \epsilon) \leq-\frac{(\lambda(1-\delta))^{1 / \alpha}}{C_{X}^{\prime \prime}},
$$

and the upper bound follows by taking $\delta \rightarrow 0$ and $\lambda \rightarrow 1$.

By choosing $K_{X}=\max \left(C_{X}^{\prime}, C_{X}^{\prime \prime}\right)$, we complete the proof of Theorem 2.1. 
Remark 3.1. When $X$ and $Y$ are independent, there is a simple proof without using the correlation inequality in the spirit of [5]. A direct proof of Corollary 2.2 can also be done as in [9].

\section{Proof of Theorem 2.4, Part I}

To simplify the reading of our paper, we introduce the following notation. Set $a_{t}=f(t) / t^{\gamma}$ and $b_{t}=\phi\left(a_{t}\right)$.

Suppose here that, with probability $1, f(t) \leq Y(t)$ for all $t$ large enough. We want to prove that $\lim _{t \rightarrow+\infty} a_{t}=0$ and $\int_{0}^{\infty} a_{t}^{-1 / \gamma} b_{t}(d t / t)<+\infty$.

In the sequel, there is no loss of generality to assume that $f(t)$ is a continuous function of $t \geq 0$.

Lemma 4.1. One has

$$
\lim _{t \rightarrow+\infty} a_{t}=0
$$

To prove Theorem 2.4, we will show that $f \in \operatorname{LUC}(Y)$ when $\int_{0}^{\infty} a_{t}^{-1 / \gamma} b_{t}(d t / t)=+\infty$ and $\lim _{t \rightarrow+\infty} a_{t}=0$.

Following the same lines as those in [11], our aim is to construct a suitable subset $J$ of $\mathbb{N}$ such that we have the following property for an appropriate family of sets $\left(E_{i}\right)_{i \in J}$ in a basic probability space: given $\epsilon>0$, there exist a number $K$ and an integer $p$ such that

$$
\forall n \in J, \quad n \geq p \Longrightarrow \sum_{m \in J, m>n} \mathbb{P}\left(E_{n} \cap E_{m}\right) \leq \mathbb{P}\left(E_{n}\right)\left(K+(1+\epsilon) \sum_{m \in J, m>n} \mathbb{P}\left(E_{m}\right)\right) .
$$

Lemma 4.2. When $\int_{0}^{\infty} a_{t}^{-1 / \gamma} b_{t}(d t / t)=+\infty$ and $\lim _{t \rightarrow+\infty} a_{t}=0$, one can find a sequence $\left\{t_{n}, n \geq 1\right\}$ with the two following properties:

$$
t_{n+1} \geq t_{n}\left(1+a_{t_{n}}^{1 / \gamma}\right), \quad \sum_{n=1}^{\infty} b_{t_{n}}=+\infty
$$

Remark 4.3. The condition " $f(t) / t^{\gamma}$ is a nonincreasing function of $t>0$ " is essential to prove Lemma 4.2 (see [7, page 373]).

To continue the construction of the set $J$, we need the following definition and notation.

Definition 4.4. Consider the interval $A_{k}=\left[2^{k}, 2^{k+1}\left[, k \in \mathbb{N}\right.\right.$. If $a_{t_{i}}^{-1 / \gamma} \in A_{k}, i \in \mathbb{N}^{*}$, then one notes $u(i)=k$.

Next, set $I_{k}=\left\{i \in \mathbb{N}^{*}, u(i)=k \in \mathbb{N}\right\}$ which is finite by Lemma 4.1 and

$$
N_{k}=\exp \left(K_{0}(\gamma \log 2)^{\beta} k^{\beta} 2^{\gamma(k-1) / \alpha}\right),
$$

where $K_{0}$ was defined in Corollary 2.2. 
Notation

(i) $U_{m, k}=\left\{i \in \mathbb{N}^{*}, i \in I_{k}, i<m\right.$, card $\left(I_{k} \cap\left[i, m[) \leq N_{k}\right\}, m \in \mathbb{N}^{*}, k \in \mathbb{N}\right.$;

(ii) $k_{0}=\inf \left\{n \in \mathbb{N}, 2^{\gamma n / \alpha} \geq 2^{\gamma / \alpha} /\left(K_{0}\left(2^{\gamma / \alpha}-1\right)(\gamma \log 2)^{\beta}\right)+2^{2 \gamma / \alpha} / K_{0}^{2}\left(2^{\gamma / \alpha}-1\right)\right\},\left(k_{0}\right.$ depends on $\gamma, \alpha, \beta$ and $K_{0}$ only);

(iii) $V_{m}=\bigcup_{k \in \mathbb{N}} U_{m, k}$, where $m$ is fixed, $u(m)=k_{1}$, and $k \geq k_{1}+k_{0}$;

(iv) $W=\bigcup_{m \geq 1} V_{m}$.

Now, we can define the set $J$ as follows:

$$
J=\mathbb{N}^{*}-W
$$

Since it is assumed that $f(t) / t^{r}$ is a nonincreasing function of $t>0$ (being a particular case of the condition " $f(t) / t^{\gamma}$ is bounded"),

$$
i<m \Longrightarrow k=u(i) \leq u(m)=k_{1}
$$

Moreover, since $k_{0} \geq 1$, we get $k<k_{1}+k_{0}$. Hence $V_{m}$ is always an empty set (by construction). Thus we obtain $J=\mathbb{N}^{*}$.

We have, by Lemma 4.2,

$$
\sum_{n \in J} b_{t_{n}}=+\infty
$$

Lemma 4.5. $n \in J, m \in J, n<m$, such that card $\left(I_{u(m)} \cap[n, m]\right)>\exp \left(K_{0} 2^{u(m)-1}\right)$, one has

$$
\frac{t_{m}}{t_{n}} \geq \exp \left(\exp \left(\frac{K_{0}}{4} 2^{\min (u(n), u(m))}\right)\right)
$$

Proof. Set $k=u(n), k_{1}=u(m)$ and $G=I_{k_{1}} \cap[n, m]=\left\{i_{1}, i_{2}, \ldots, i_{z}\right\}$, where $n \leq i_{1}<i_{2}<\cdots<$ $i_{z} \leq m$. We have

$$
\frac{t_{m}}{t_{n}}=\frac{t_{m}}{t_{i_{z}}} \frac{t_{i_{z}}}{t_{i_{z-1}}} \cdots \frac{t_{i_{1}}}{t_{n}}
$$

Note that, when $i \in I_{k_{1}}$, we have $t_{i+1} \geq t_{i}\left(1+a_{t_{i}}^{1 / \gamma}\right) \geq t_{i}\left(1+2^{-k_{1}-1}\right)$. Moreover, since card $(G)>\exp \left(K_{0} 2^{k_{1}-1}\right)$ by hypothesis, (4.9) implies

$$
\frac{t_{m}}{t_{n}} \geq \exp \left(\exp \left(K_{0} 2^{k_{1}-1}\right) \log \left(1+2^{-k_{1}-1}\right)\right) \geq \exp \left(\exp \left(\frac{K_{0}}{4} 2^{k_{1}}\right)\right),
$$

when $n$ hence $k_{1}$ are large enough.

Thus, since $k \leq k_{1},(4.10)$ implies (4.8).

The proof of Lemma 4.5 is now complete. 


\section{Proof of Theorem 2.4, Part II}

Consider, now, the events $E_{n}=\left\{Y\left(t_{n}\right)<f\left(t_{n}\right)\right\}$. We have directly $\mathbb{P}\left(E_{n}\right)=b_{t_{n}}$, and, by (4.7), $\sum_{n \in J} b_{t_{n}}=+\infty$. To prove (4.2), we remark that, given $n \in J, J$ can be rewritten as follows: $J=J^{\prime} \cup\left(\bigcup_{k \in \mathbb{N}} J_{k}\right) \cup J^{\prime \prime}$, where $J^{\prime}=\left\{m \in J, t_{n} \leq t_{m} \leq 2 t_{n}\right\}, J_{k}=\left\{m \in J \cap I_{k}, t_{m}>2 t_{n}\right.$, card $\left(I_{k} \cap\right.$ $\left.[n, m]) \leq \exp \left(K_{0} 2^{k-1}\right)\right\}$, and $J^{\prime \prime}=J-\left(J^{\prime} \cup\left(\bigcup_{k \in \mathbb{N}} J_{k}\right)\right)$.

Our first key small ball estimate is given in the following lemma.

Lemma 5.1. Consider $0<t<u$, and $\theta, v>0$. Then one has

$$
\mathbb{P}\left(\left\{Y(t) \leq \theta t^{\gamma}\right\} \cap\{Y(u) \leq v\}\right) \leq \exp \left(K_{5}\right) \mathbb{P}\left(Y(t) \leq \theta t^{\gamma}\right) \exp \left(-\frac{K_{5}(u-t)}{v^{1 / \gamma}}\right),
$$

where $K_{5}$ depends on $H_{1}, H_{2}, H_{3}, H_{4}, \lambda_{1}$, and $\lambda_{2}$ only.

Proof. Set $F_{1}=\left\{Y(t) \leq \theta t^{r}\right\}$ and $F_{2}=\{Y(u) \leq v\}$. We have

$$
\begin{aligned}
\mathbb{P}\left(F_{1} \cap F_{2}\right) & =\mathbb{P}\left(F_{1} \cap\left\{\sup _{0 \leq s \leq u} \sup _{0 \leq w_{1}, w_{2}, w_{3}, w_{4} \leq s}\left|X\left(w_{1}, w_{2}, w_{3}, w_{4}, s\right)\right| \leq v\right\}\right) \\
& \leq \mathbb{P}\left(F_{1} \cap\left\{\sup _{t \leq s \leq u} \sup _{0 \leq w_{1}, w_{2}, w_{3}, w_{4} \leq s}\left|X\left(w_{1}, w_{2}, w_{3}, w_{4}, s\right)\right| \leq v\right\}\right) \\
& \leq \mathbb{P}\left(F_{1} \cap\left\{\sup _{t \leq s \leq u} \sup _{0 \leq w_{1}, w_{2} \leq s}\left|\lambda_{1} s^{H_{3}+H_{4}} B_{H_{1}, H_{2}}\left(w_{1}, w_{2}\right)\right| \leq v\right\}\right) .
\end{aligned}
$$

Denote, by $[x]$, the integer part of a real $x$. Let $\delta>0$. We consider the sequence $t_{k}, k \in$ $\{0, \ldots, n\}$, where $t_{0}=t, t_{k+1}=t_{k}+\delta$, and $n=[(u-t) / \delta]$. Consider also the rectangles $R_{j}=$ $\left[t_{j}, t_{j+1}\right] \times\left[t_{j}, t_{j+1}\right]$, where $j \in\{0, \ldots, n-1\}$. Their area is $\left(t_{j+1}-t_{j}\right)^{2}=\delta^{2}$. Let $G_{j}$ be the event defined by

$$
G_{j}=F_{1} \cap\left\{\sup _{t \leq s \leq t_{j}} \sup _{0 \leq w_{1}, w_{2} \leq s}\left|\lambda_{1} s^{H_{3}+H_{4}} B_{H_{1}, H_{2}}\left(w_{1}, w_{2}\right)\right| \leq v\right\} .
$$

We have $F_{1} \cap F_{2} \subset G_{j}$.

Moreover, we have also

$$
G_{j+1} \subset G_{j} \cap\left\{Z_{j} \leq 4 v\right\}
$$

where

$$
Z_{j}=\lambda_{1} t_{j+1}^{H_{3}+H_{4}}\left(B_{H_{1}, H_{2}}\left(t_{j+1}, t_{j+1}\right)-B_{H_{1}, H_{2}}\left(t_{j}, t_{j+1}\right)-B_{H_{1}, H_{2}}\left(t_{j+1}, t_{j}\right)+B_{H_{1}, H_{2}}\left(t_{j}, t_{j}\right)\right) .
$$
by

Before rewritting $Z_{j}$, we recall the integral representation of $B_{H_{k}, H_{l}}, k \in \mathbb{N}^{*}, l \in \mathbb{N}^{*}$, given

$$
B_{H_{k}, H_{l}}\left(s_{k}, s_{l}\right)=\int_{-\infty}^{s_{k}} \int_{-\infty}^{s_{l}} g_{H_{k}}\left(s_{k}, u_{k}\right) g_{H_{l}}\left(s_{l}, u_{l}\right) W\left(d\left(u_{k}, u_{l}\right)\right)
$$


where $W\left(u_{k}, u_{l}\right), u_{k} \in \mathbb{R}, u_{l} \in \mathbb{R}$, is a standard Brownian sheet, $g_{H}(s, u)=k_{2 H}^{-1}(\max (s$ $\left.-u, 0)^{H-1 / 2}-\max (-u, 0)^{H-1 / 2}\right)$, and $k_{2 H}$ is a normalizing constant.

Hence $Z_{j}$ can be rewritten by (5.6) as follows: $Z_{j}=Z_{j, 1}+Z_{j, 2}$, where

$$
Z_{j, 1}=\lambda_{1} k_{2 H_{1}}^{-1} k_{2 H_{2}}^{-1} t_{j+1}^{H_{3}+H_{4}} \int_{t_{j}}^{t_{j+1}} \int_{t_{j}}^{t_{j+1}}\left(t_{j+1}-u_{1}\right)^{H_{1}-1 / 2}\left(t_{j+1}-u_{2}\right)^{H_{2}-1 / 2} W\left(d\left(u_{1}, u_{2}\right)\right) .
$$

Note also that $Z_{j, 1}$ and $Z_{j, 2}$ are independent.

Since $\mathbb{P}\left(\left|Z_{j, 1}+x\right| \leq 4 v\right)$ is maximum at $x=0$ and $Z_{j, 1}$ and $G_{j}$ are independent, we have

$$
\mathbb{P}\left(G_{j+1}\right) \leq \mathbb{P}\left(G_{j}\right) \mathbb{P}\left(\left|Z_{j, 1}\right| \leq 4 v\right) .
$$

The integral representation of $Z_{j, 1}$ implies that $\mathbb{E}\left(Z_{j, 1}\right)=0$ and

$$
\operatorname{Var} Z_{j, 1}=\frac{\lambda_{1}^{2} k_{2 H_{1}}^{-2} k_{2 H_{2}}^{-2}}{4 H_{1} H_{2}} \delta^{2\left(H_{1}+H_{2}\right)} t_{j+1}^{2\left(H_{3}+H_{4}\right)} \geq \frac{\lambda_{1}^{2} k_{2 H_{1}}^{-2} k_{2 H_{2}}^{-2}}{4 H_{1} H_{2}} \delta^{2 \gamma}:=L^{2} \delta^{2 \gamma} .
$$

Denote, by $\Phi$, the distribution function of the absolute value of a standard Gaussian random variable. Then we obtain

$$
\mathbb{P}\left(Z_{j+1}\right) \leq \mathbb{P}\left(Z_{j}\right) \Phi\left(\frac{4 v}{L \delta^{\gamma}}\right),
$$

and therefore, $\mathbb{P}\left(F_{1} \cap F_{2}\right) \leq \mathbb{P}\left(F_{1}\right) \Phi\left(4 v / L \delta^{r}\right)^{n}$.

Choosing $\delta=v^{1 / \gamma}$, we get $K_{5}=-\log \Phi(2 / L)$. Lemma 5.1 is proved.

Lemma 5.2. $\sum_{m \in J^{\prime}} \mathbb{P}\left(E_{n} \cap E_{m}\right) \leq K^{\prime} b_{t_{n}}$ and $\sum_{m \in\left(\cup_{k} J_{k}\right)} \mathbb{P}\left(E_{n} \cap E_{m}\right) \leq K^{\prime \prime} b_{t_{n}}$, where $K^{\prime}$ and $K^{\prime \prime}$ are numbers.

Proof. Setting $u=t_{m}, t=t_{n}, \theta=a_{t_{n}}$ and $v=f\left(t_{m}\right)$, Lemma 5.1 implies

$$
\mathbb{P}\left(E_{n} \cap E_{m}\right) \leq \exp \left(K_{5}\right) b_{t_{n}} \exp \left(-\frac{K_{5}\left(t_{m}-t_{n}\right)}{f\left(t_{m}\right)^{1 / \gamma}}\right) .
$$

Consider, first, the case when $m \in J^{\prime}$.

Lemma 4.2 implies that, for all $i \geq n$, we have $t_{i+1}-t_{i} \geq t_{i} a_{t_{i}}^{1 / \gamma}=f\left(t_{i}\right)^{1 / \gamma} \geq f\left(t_{n}\right)^{1 / \gamma}$. Then we can establish

$$
t_{m}-t_{n} \geq(m-n) f\left(t_{n}\right)^{1 / \gamma}, \quad f\left(t_{m}\right) \leq f\left(t_{n}\right)\left(\frac{t_{m}}{t_{n}}\right)^{\gamma} \leq 2^{\gamma} f\left(t_{n}\right)
$$

Combining (5.11) with (5.12), we get

$$
\mathbb{P}\left(E_{n} \cap E_{m}\right) \leq \exp \left(K_{5}\right) b_{t_{n}} \exp \left(-\frac{K_{5}(m-n)}{2}\right),
$$

which is the first part of Lemma 5.2.

Consider, now, the case $m \in J_{k}$. 
Combining (5.11) with the definition of $J_{k}$, we have

$$
\mathbb{P}\left(E_{n} \cap E_{m}\right) \leq \exp \left(K_{5}\right) b_{t_{n}} \exp \left(-\frac{K_{5}}{2\left(a_{t_{m}}\right)^{1 / \gamma}}\right) .
$$

Since $u(m)=k$, we get

$$
\mathbb{P}\left(E_{n} \cap E_{m}\right) \leq \exp \left(K_{5}\right) b_{t_{n}} \exp \left(-K_{5} 2^{k-1}\right),
$$

and consequently, by noting that card $J_{k} \leq \operatorname{card}\left(I_{k} \cap[n, m]\right) \leq \exp \left(K_{0} 2^{k-1}\right)$ and by assuming $K_{0}<K_{5}$, we have

$$
\sum_{m \in J_{k}} \mathbb{P}\left(E_{n} \cap E_{m}\right) \leq \exp \left(K_{5}\right) b_{t_{n}} \exp \left(\left(K_{0}-K_{5}\right) 2^{k-1}\right) .
$$

Lemma 5.2 is, therefore, proved.

To deal with the set $J^{\prime \prime}$, we first state a standard large deviation result and a technical lemma (see [6]).

Lemma A. Let $X=\left\{X\left(s_{1}, s_{2}\right),\left(s_{1}, s_{2}\right) \in[0,1]^{2}\right\}$ be a separable real-valued centered Gaussian process such that $X(0,0)=0$ with probability 1 and satisfying, for any $\left[s_{1}, s_{1}+h_{1}\right] \times\left[s_{2}, s_{2}+h_{2}\right] \subset[0,1]^{2}$,

$$
\left(\mathbb{E X}\left(\left[s_{1}, s_{1}+h_{1}\right] \times\left[s_{2}, s_{2}+h_{2}\right]\right)^{2}\right)^{1 / 2} \leq \kappa\left(h_{1}, h_{2}\right) \leq c_{\kappa} h_{1}^{\alpha_{1}} h_{2}^{\alpha_{2}}, \quad \alpha_{1}>0, \alpha_{2}>0,
$$

where

$$
X\left(\left[s_{1}, t_{1}\right] \times\left[s_{2}, t_{2}\right]\right)=\int_{\left[s_{1}, t_{1}\right] \times\left[s_{2}, t_{2}\right]} X\left(d\left(u_{1}, u_{2}\right)\right) .
$$

Then one has, for $c_{\kappa}^{-1} \delta>1$,

$$
\mathbb{P}\left(\sup _{\left(s_{1}, s_{2}\right) \in[0,1]^{2}}\left|X\left(s_{1}, s_{2}\right)\right| \geq \delta\right) \leq \frac{1}{C} \exp \left(-C\left(c_{\mathcal{K}}^{-1} \delta\right)^{2}\right),
$$

where $C$ is a positive constant independent of $c_{\kappa}$ and $\delta$.

Lemma B. One has, for $\epsilon_{1}>\epsilon / 2$, where $\epsilon$ is small enough,

$$
\exp \left(-K_{3} \frac{\left|\epsilon_{1}-\epsilon\right|(\log (1 / \epsilon))^{\beta}}{\epsilon^{1+1 / \alpha}}\right) \leq \frac{\phi\left(\epsilon_{1}\right)}{\phi(\epsilon)} \leq \exp \left(K_{3} \frac{\left|\epsilon_{1}-\epsilon\right|(\log (1 / \epsilon))^{\beta}}{\epsilon^{1+1 / \alpha}}\right),
$$

where $K_{3}>0$.

Building on Lemmas A and B, we can establish our last key small ball estimate in the following result. 
Lemma 5.3. Let $\lambda$ be a real number such that $1 / 2<\lambda<1$. Set

$$
r=\min \left(\frac{1-\max \left(H_{1}, H_{2}, H_{3}, H_{4}\right)}{3}, \frac{(1-\lambda) \alpha}{3}\right) .
$$

Then one has, for $u \geq 2 t$,

$$
\begin{aligned}
\mathbb{P}(Y(t) \leq & \left.\theta t^{\gamma}, Y(u) \leq v u^{\gamma}\right) \\
\leq & \phi(\theta) \phi(v) \exp \left(2\left(\frac{t}{u}\right)^{r} K_{3}\left(\frac{(\log (1 / \theta))^{\beta}}{\theta^{1+(1 / \alpha)}}+\frac{(\log (1 / v))^{\beta}}{v^{1+(1 / \alpha)}}\right)\right) \\
& +3\left(\frac{1}{C_{12,2}} \exp \left(-\frac{C_{12,2}}{4 \lambda_{1}^{2} K_{H_{1}, 2}^{2}}\left(\frac{u}{t}\right)^{r}\right)+\frac{1}{C_{34,2}} \exp \left(-\frac{C_{34,2}}{4 \lambda_{2}^{2} K_{H_{3,2}}^{2}}\left(\frac{u}{t}\right)^{r}\right)\right) \\
& +3\left(\frac{1}{C_{12,1}} \exp \left(-\frac{C_{12,1}}{4 \lambda_{1}^{2} K_{H_{1}, 1}^{2}}\left(\frac{u}{t}\right)^{r}\right)+\frac{1}{C_{34,1}} \exp \left(-\frac{C_{34,1}}{4 \lambda_{2}^{2} K_{H_{3}, 1}^{2}}\left(\frac{u}{t}\right)^{r}\right)\right),
\end{aligned}
$$

where $K_{H_{1}, 1}, K_{H_{1}, 2}>0$ depend on $H_{1}\left(H_{1} \leq H_{2}\right)$ only, $K_{H_{3}, 1}, K_{H_{3}, 2}>0$ depend on $H_{3}\left(H_{3} \leq H_{4}\right)$ only, $K_{3}>0$ is defined as in Lemma $B$, and $C_{12,1}, C_{12,2}, C_{34,1}, C_{34,2}>0$ are defined as in Lemma $A$.

Proof. Set $Q=\mathbb{P}\left(Y(t) \leq \theta t^{\gamma}, Y(u) \leq v u^{\gamma}\right)$.

Set $v=\sqrt{u t}$. If $t=o(u)$, then $t=o(v)$ and $v=o(u)$.

Based on (5.6), $B_{H_{1}, H_{2}}$ and $B_{H_{3}, H_{4}}$ can be split as follows:

$$
B_{H_{1}, H_{2}}=B_{H_{1}, H_{2}, 1}+B_{H_{1}, H_{2}, 2} \quad \quad B_{H_{3}, H_{4}}=B_{H_{3}, H_{4}, 1}+B_{H_{3}, H_{4}, 2}
$$

where we have, for $(i, j) \in\{(1,2),(3,4)\}$,

$$
B_{H_{i}, H_{j}, 1}\left(w_{i}, w_{j}\right)=\int_{\left|x_{i}\right| \leq v} \int_{-\infty}^{w_{j}} g_{H_{i}}\left(w_{i}, x_{i}\right) g_{H_{j}}\left(w_{j}, x_{j}\right) W\left(d\left(x_{i}, x_{j}\right)\right) .
$$

Note that $B_{H_{1}, H_{2}, 1}$ and $B_{H_{1}, H_{2}, 2}$ are independent as $B_{H_{3}, H_{4}, 1}$ and $B_{H_{3}, H_{4}, 2}$.

Equation (5.23) implies that the FMFBS $X$ can be rewritten as follows: $X=X_{1}+X_{2}$, where

$$
X_{i}\left(w_{1}, w_{2}, w_{3}, w_{4}, s\right)=\lambda_{1} s^{H_{3}+H_{4}} B_{H_{1}, H_{2}, i}\left(w_{1}, w_{2}\right)+\lambda_{2} s^{H_{1}+H_{2}} B_{H_{3}, H_{4}, i}\left(w_{3}, w_{4}\right) .
$$

Set

$$
Y_{i}(t)=\sup _{0 \leq s \leq t} \sup _{0 \leq w_{1}, w_{2}, w_{3}, w_{4} \leq s}\left|X_{i}\left(w_{1}, w_{2}, w_{3}, w_{4}, s\right)\right|, \quad t \geq 0, i \in\{1,2\} .
$$

Then, given $\delta>0$, we have (see [11])

$$
Q \leq \phi(\theta+2 \delta) \phi(v+2 \delta)+3 \mathbb{P}\left(Y_{2}(t)>\delta t^{\gamma}\right)+3 \mathbb{P}\left(Y_{1}(u)>\delta u^{\gamma}\right) .
$$

Equation (5.20) implies

$$
\phi(\theta+2 \delta) \leq \phi(\theta) \exp \left(2 \delta K_{3}\left(\frac{(\log (1 / \theta))^{\beta}}{\theta^{1+(1 / \alpha)}}\right)\right),
$$


and, consequently,

$$
\phi(\theta+2 \delta) \phi(v+2 \delta) \leq \phi(\theta) \phi(v) \exp \left(2 \delta K_{3}\left(\frac{(\log (1 / \theta))^{\beta}}{\theta^{1+(1 / \alpha)}}+\frac{(\log (1 / v))^{\beta}}{v^{1+(1 / \alpha)}}\right)\right) .
$$

If we choose $\delta=(t / u)^{r}$, then we get the first term of the RHS of Lemma 5.3.

Next, we want to obtain an upper bound of

$$
\begin{aligned}
& \mathbb{P}\left(Y_{2}(t)>\delta t^{\gamma}\right) \\
& \quad=\mathbb{P}\left(\sup _{0 \leq s \leq 1} \sup _{0 \leq w_{1}, w_{2}, w_{3}, w_{4} \leq s}\left|\lambda_{1} s^{H_{3}+H_{4}} L_{H_{1}, H_{2}, 2}\left(w_{1}, w_{2}\right)+\lambda_{2} s^{H_{1}+H_{2}} L_{H_{3}, H_{4}, 2}\left(w_{3}, w_{4}\right)\right|>\delta\right),
\end{aligned}
$$

where we have, for $(i, j) \in\{(1,2),(3,4)\}$,

$$
L_{H_{i}, H_{j}, 2}\left(w_{i}, w_{j}\right)=\int_{\left|x_{i}\right| \geq v / t} \int_{-\infty}^{w_{j}} g_{H_{i}}\left(w_{i}, x_{i}\right) g_{H_{j}}\left(w_{j}, x_{j}\right) W\left(d\left(x_{i}, x_{j}\right)\right) .
$$

We can show, by standard computations, that

$$
\begin{aligned}
\mathbb{P}\left(Y_{2}(t)>\delta t^{r}\right) \leq & \mathbb{P}\left(\sup _{0 \leq w_{1}, w_{2} \leq 1}\left|L_{H_{1}, H_{2}, 2}\left(w_{1}, w_{2}\right)\right|>\frac{\delta}{2\left|\lambda_{1}\right|}\right) \\
& +\mathbb{P}\left(\sup _{0 \leq w_{3}, w_{4} \leq 1}\left|L_{H_{3}, H_{4}, 2}\left(w_{3}, w_{4}\right)\right|>\frac{\delta}{2\left|\lambda_{2}\right|}\right) .
\end{aligned}
$$

Denote, by $\sigma_{H}$, the covariance function of a FBM $B_{H}$. Set $\sigma_{H, 2}$, the covariance function of the process $\left\{B_{H, 2}(w), 0 \leq w \leq 1\right\}$, defined by

$$
B_{H, 2}(w)=\int_{|x| \geq v / t} g_{H}(w, x) \widetilde{W}(d x)
$$

where $\widetilde{W}(x), x \in \mathbb{R}$, is a Wiener process.

Since

$$
\mathbb{E}\left(L_{H_{1}, H_{2}, 2}\left(w_{1}, w_{2}\right) L_{H_{1}, H_{2}, 2}\left(w_{1}^{\prime}, w_{2}^{\prime}\right)\right)=\sigma_{H_{1}, 2}\left(w_{1}, w_{1}^{\prime}\right) \times \sigma_{H_{2}}\left(w_{2}, w_{2}^{\prime}\right),
$$

we have, for any $\left[w_{1}, w_{1}+h_{1}\right] \times\left[w_{2}, w_{2}+h_{2}\right] \subset[0,1]^{2}$,

$$
\begin{aligned}
\mathbb{E}\left(L_{H_{1}, H_{2}, 2}\left(\left[w_{1}, w_{1}+h_{1}\right] \times\left[w_{2}, w_{2}+h_{2}\right]\right)^{2}\right) \\
=\mathbb{E}\left(\int_{\left[w_{1}, w_{1}+h_{1}\right] \times\left[w_{2}, w_{2}+h_{2}\right]} L_{H_{1}, H_{2}, 2}\left(d\left(x_{1}, x_{2}\right)\right)\right. \\
\left.\quad \times \int_{\left[w_{1}, w_{1}+h_{1}\right] \times\left[w_{2}, w_{2}+h_{2}\right]} L_{H_{1}, H_{2}, 2}\left(d\left(x_{1}^{\prime}, x_{2}^{\prime}\right)\right)\right) \\
\leq \int_{w_{1}}^{w_{1}+h_{1}} \int_{w_{1}}^{w_{1}+h_{1}}\left|\sigma_{H_{1}, 2}\left(x_{1}, x_{1}^{\prime}\right)\right| d x_{1} d x_{1}^{\prime} \\
\quad \times \int_{w_{2}}^{w_{2}+h_{2}} \int_{w_{2}}^{w_{2}+h_{2}}\left|\sigma_{H_{2}}\left(x_{2}, x_{2}^{\prime}\right)\right| d x_{2} d x_{2}^{\prime}:=I \times I I .
\end{aligned}
$$


Consider II first. We get, by the inequality of Cauchy-Schwarz,

$$
I I \leq \int_{w_{2}}^{w_{2}+h_{2}} \int_{w_{2}}^{w_{2}+h_{2}} x_{2}^{H_{2}} x_{2}^{\prime H_{2}} d x_{2} d x_{2}^{\prime} \leq h_{2}^{2}
$$

Consider I now.

A straight computation implies that there exists $K_{H_{1,2}}>0$ depending on $H_{1}$ such that

$$
\mathbb{E}\left(B_{H_{1}, 2}\left(x_{1}\right)\right)^{2} \leq K_{H_{1}, 2}^{2} x_{1}^{2}(v / t)^{2 H_{1}-2},
$$

and, consequently, by the inequality of Cauchy-Schwarz,

$$
\left|\sigma_{H_{1}, 2}\left(x_{1}, x_{1}^{\prime}\right)\right| \leq K_{H_{1}, 2}^{2} x_{1} x_{1}^{\prime}(v / t)^{2 H_{1}-2} .
$$

So we get

$$
I \leq K_{H_{1}, 2}^{2}(v / t)^{2 H_{1}-2} h_{1}^{2}
$$

Hence, combining (5.35) with (5.36) and (5.39), we have

$$
\mathbb{E}\left(L_{H_{1}, H_{2}, 2}\left(\left[w_{1}, w_{1}+h_{1}\right] \times\left[w_{2}, w_{2}+h_{2}\right]\right)^{2}\right) \leq K_{H_{1}, 2}^{2}(v / t)^{2 H_{1}-2} h_{1}^{2} h_{2}^{2} .
$$

An application of Lemma A with $\alpha_{1}=\alpha_{2}=1, c_{\mathcal{K}}=K_{H_{1}, 2}(v / t)^{H_{1}-1}$, and $c_{\mathcal{K}}^{-1} \delta>1$ implies that

$$
\mathbb{P}\left(\sup _{0 \leq w_{1}, w_{2} \leq 1}\left|L_{H_{1}, H_{2,2}}\left(w_{1}, w_{2}\right)\right|>\frac{\delta}{2\left|\lambda_{1}\right|}\right) \leq \frac{1}{C_{12,2}} \exp \left(-\frac{C_{12,2}}{K_{H_{1,2}}^{2}\left(v^{2} / t^{2}\right)^{H_{1}-1}} \frac{\delta^{2}}{4 \lambda_{1}^{2}}\right) .
$$

Similarly, we can establish

$$
\mathbb{P}\left(\sup _{0 \leq w_{3}, w_{4} \leq 1}\left|L_{H_{3}, H_{4}, 2}\left(w_{3}, w_{4}\right)\right|>\frac{\delta}{2\left|\lambda_{2}\right|}\right) \leq \frac{1}{C_{34,2}} \exp \left(-\frac{C_{34,2}}{K_{H_{3,2}}^{2}\left(v^{2} / t^{2}\right)^{H_{3}-1}} \frac{\delta^{2}}{4 \lambda_{2}^{2}}\right) .
$$

Set $\delta=(t / u)^{r}$. Recall that $v^{2}=u t$ and $r \leq\left(1-\max \left(H_{1}, H_{2}, H_{3}, H_{4}\right)\right) / 3$. Combining (5.32) with (5.41) and (5.42), we get

$$
\mathbb{P}\left(Y_{2}(t)>\delta t^{r}\right) \leq \frac{1}{C_{12,2}} \exp \left(-\frac{C_{12,2}}{4 \lambda_{1}^{2} K_{H_{1,2}}^{2}}\left(\frac{u}{t}\right)^{r}\right)+\frac{1}{C_{34,2}} \exp \left(-\frac{C_{34,2}}{4 \lambda_{2}^{2} K_{H_{3}, 2}^{2}}\left(\frac{u}{t}\right)^{r}\right)
$$

that is the second term of the RHS of Lemma 5.3. 
Finally, we can establish a similar result for $\mathbb{P}\left(Y_{1}(u)>\delta u^{\gamma}\right)$, that is,

$$
\mathbb{P}\left(Y_{1}(u)>\delta u^{r}\right) \leq \frac{1}{C_{12,1}} \exp \left(-\frac{C_{12,1}}{4 \lambda_{1}^{2} K_{H_{1}, 1}^{2}}\left(\frac{u}{t}\right)^{r}\right)+\frac{1}{C_{34,1}} \exp \left(-\frac{C_{34,1}}{4 \lambda_{2}^{2} K_{H_{3}, 1}^{2}}\left(\frac{u}{t}\right)^{r}\right),
$$

which achieves the proof of Lemma 5.3.

Finally, we state the last technical lemma.

Lemma 5.4. There exists an integer $p$ such that if $n>\sup _{s \leq p}\left(\sup I_{s}\right)$, then for $m \in J^{\prime \prime}, m>n$, given $\epsilon>0$, one has $\mathbb{P}\left(E_{n} \cap E_{m}\right) \leq(1+\epsilon) b_{t_{n}} b_{t_{m}}$.

Proof. Let $u(n)=k^{\prime}$ and $u(m)=k_{1}$. We have, by Lemma 4.5,

$$
\frac{t_{m}}{t_{n}} \geq \exp \left(\exp \left(\frac{K_{0}}{4} 2^{\min \left(k^{\prime}, k_{1}\right)}\right)\right) .
$$

Let $p \in \mathbb{N}$. Then $k^{\prime}>p$ and $k_{1}>p$. Thus we have $\min \left(k^{\prime}, k_{1}\right)>p$.

Set $t=t_{n}, u=t_{m}, \theta=a_{t_{n}}$ and $v=a_{t_{m}}$. Note that $\log (1 / \theta) \leq 1 / \theta \leq 2^{\left(k^{\prime}+1\right) \alpha}, \log (1 / v) \leq$ $1 / v \leq 2^{\left(k_{1}+1\right) \alpha}$, and $1 / b_{t_{n}} b_{t_{m}}=\exp (\psi(\theta)+\psi(v))$.

By using Lemma 5.3 and letting $p \rightarrow+\infty$, we end the proof of Lemma 5.4.

Lemmas 5.2 and 5.4 yield that (4.2) holds. Combining Borel-Cantelli's second lemma with (4.2) and (4.7), we show that, given $\epsilon>0$,

$$
\sum_{n \in J} \mathbb{P}\left(E_{n}\right) \geq \frac{1+2 K}{\epsilon} \Longrightarrow \frac{1}{1+2 \epsilon} \leq \mathbb{P}\left(\bigcup_{n \in J} E_{n}\right)=\mathbb{P}\left(\bigcup_{n \in J}\left\{Y\left(t_{n}\right) \leq f\left(t_{n}\right)\right\}\right),
$$

and, consequently, $f \in \operatorname{LUC}(Y)$. The proof of Theorem 2.4 is now complete.

\section{Acknowledgment}

The author thanks the referee for the insightful comments.

\section{References}

[1] W. V. Li and Q.-M. Shao, "Gaussian processes: inequalities, small ball probabilities and applications," in Stochastic Processes: Theory and Methods, pp. 533-597, North-Holland, Amsterdam, 2001.

[2] P. Cheridito, “Mixed fractional Brownian motion," Bernoulli, vol. 7, no. 6, pp. 913-934, 2001.

[3] F. Gassmann and D. Bürki, "Experimental investigation of atmospheric dispersion over the swiss plain—experiment SIESTA," Boundary-Layer Meteorology, vol. 41, pp. 295-307, 1987.

[4] F. Gassmann, P. Gaglione, S. E. Gryning, et al., "Experimental Investigation of Atmospheric Dispersion over Complex Terrain in a Prealpine Region (experiment SIESTA)," Swiss Federal Institute for Reactor Research EIR 604, 1986.

[5] W. V. Li, "A Gaussian correlation inequality and its applications to small ball probabilities," Electronic Communications in Probability, vol. 4, pp. 111-118, 1999.

[6] C. El-Nouty, "The influence of a log-type small ball factor in the study of the lower classes," Bulletin des Sciences Mathématiques, vol. 129, no. 4, pp. 318-338, 2005.

[7] C. El-Nouty, "On the lower classes of fractional Brownian motion," Studia Scientiarum Mathematicarum Hungarica, vol. 37, no. 3-4, pp. 363-390, 2001.

[8] C. El-Nouty, "The fractional mixed fractional Brownian motion," Statistics \& Probability Letters, vol. 65, no. 2, pp. 111-120, 2003. 
[9] C. El-Nouty, "The fractional mixed fractional Brownian motion and fractional Brownian sheet," ESAIM. Probability and Statistics, vol. 11, pp. 448-465, 2007.

[10] P Révész, Random Walk in Random and Nonrandom Environments, World Scientific, Teaneck, NJ, USA, 1990.

[11] M. Talagrand, "Lower classes for fractional Brownian motion," Journal of Theoretical Probability, vol. 9, no. 1, pp. 191-213, 1996.

[12] E. Belinsky and W. Linde, "Small ball probabilities of fractional brownian sheets via fractional integration operators," Journal of Theoretical Probability, vol. 15, no. 3, pp. 589-612, 2002.

[13] D. M. Mason and Z. Shi, "Small deviations for some multi-parameter Gaussian processes," Journal of Theoretical Probability, vol. 14, no. 1, pp. 213-239, 2001.

[14] C. Borell, "Convex measures on locally convex spaces," Arkiv för Matematik, vol. 12, pp. 239-252, 1974.

[15] M. A Lifshits, Gaussian Random Functions, vol. 322, Kluwer Academic Publishers, Dordrecht, The Netherlands, 1995. 


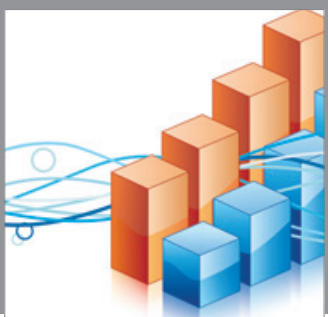

Advances in

Operations Research

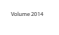

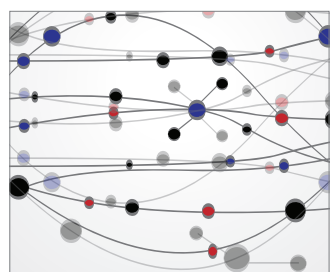

\section{The Scientific} World Journal
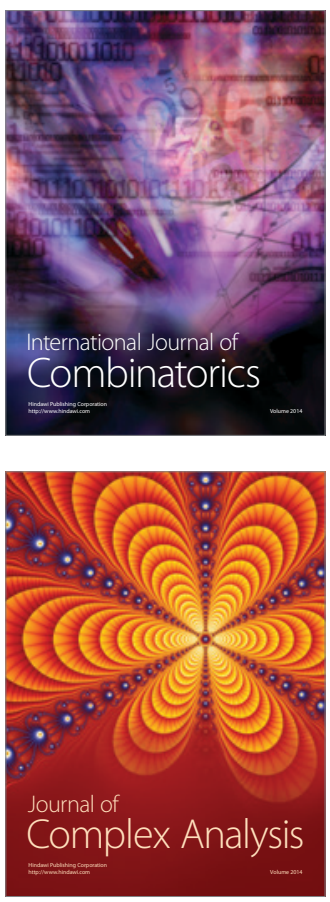

International Journal of

Mathematics and

Mathematical

Sciences
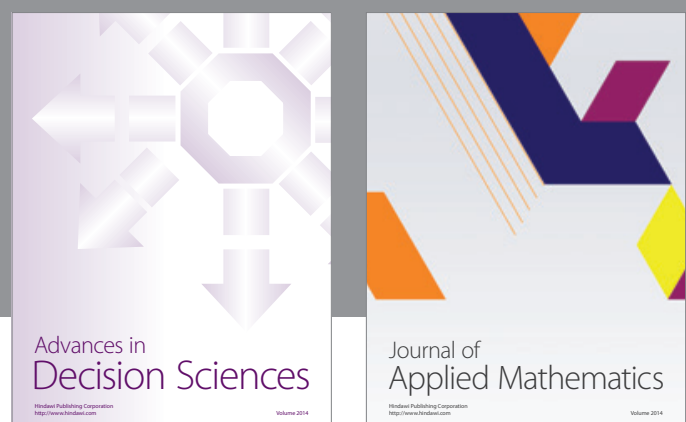

Journal of

Applied Mathematics
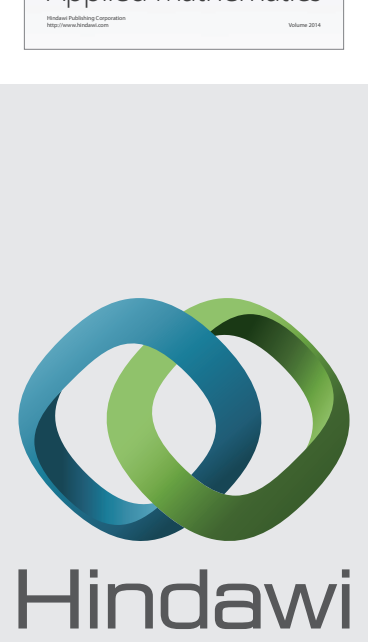

Submit your manuscripts at http://www.hindawi.com
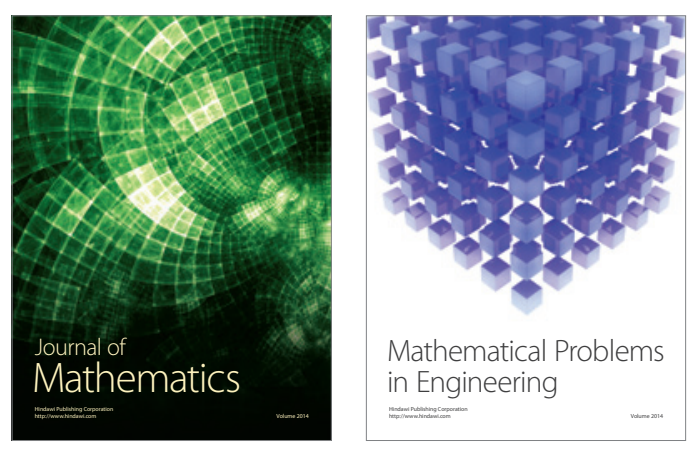

Mathematical Problems in Engineering
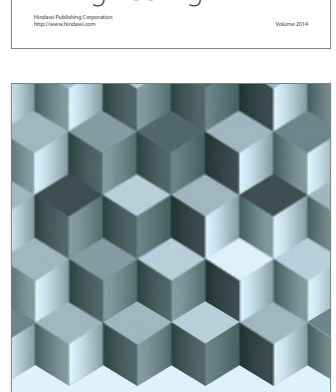

Journal of

Function Spaces
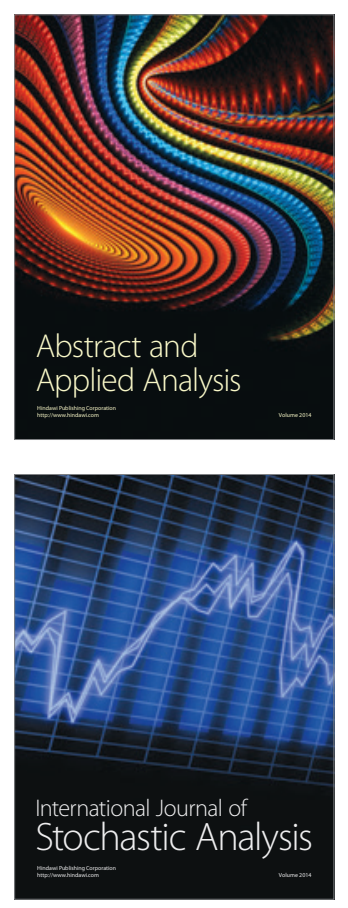

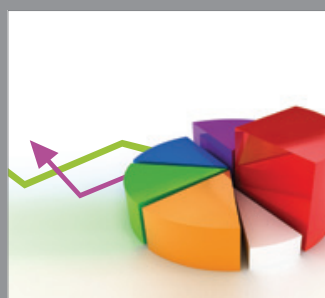

ournal of

Probability and Statistics

Promensencen
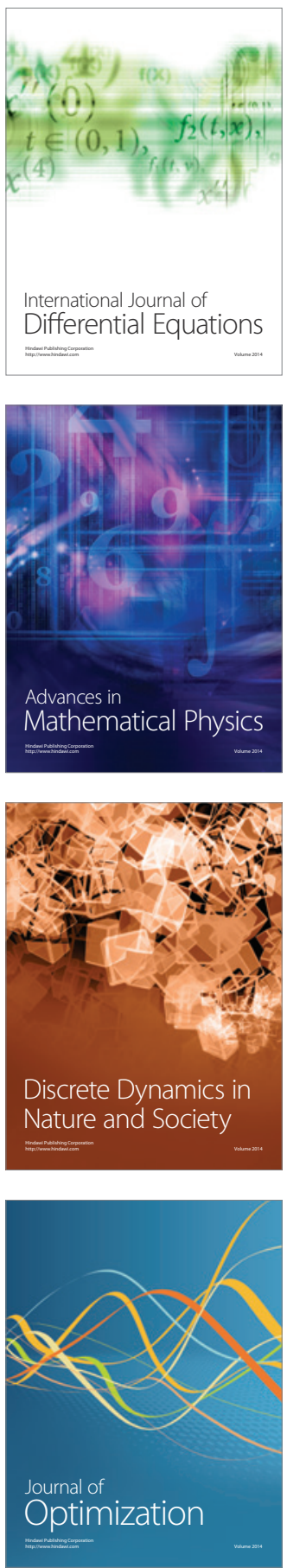